\title{
Complicated mandible fracture treatment with xenogenic bone graft: A case report
}

\author{
Diachkova E.Yu. ${ }^{*}$, Popova S.V. ${ }^{2}$, Arazashvili L.D. ${ }^{2}$, Petruk P.S. ${ }^{2}$, Cherkesov I.V. ${ }^{2}$
}

1 Department of Oral Surgery, Borovsky Institute of Dentistry, I.M. Sechenov First Moscow State Medical University (Sechenov University), 119991, Russia, 8-2 Trubetskaya str.; secu2003@mail.ru (E.D.)

2 Department of Maxillofacial Surgery, Borovsky Institute of Dentistry, I.M. Sechenov First Moscow State Medical University (Sechenov University), 119991, Russia, 8-2 Trubetskaya str.: doctorsofia@yandex.ru (P.S.); arazashvili@mail.ru (A.L.); petruk pavel@yahoo.com (P.P.); cherkesovi@gmail.com (C.I.)

* Correspondence: secu2003@mail.ru

\begin{abstract}
The problem of filling the bone cavity-forming after tooth extraction remains relevant in maxillo-facial surgery. There is a large selection of osteotropic materials of various natures for filling bone defects. In this article, our experience in the treatment of patients with combined mandible angle fracture and radicular cyst and fractures is introduced. A feature of the treatment is to fill the bone defect with the osteotropic material. Using collagen osteotropic material, possessing osteoconductive property can improve the treatment of patients with mandibular fractures within the dentition. This is due to both the stabilization of the fracture line, a decrease in the likelihood of displacement of fragments along with fixation with devices, and a reduction in the time of bone tissue regeneration, which reduces the rehabilitation period and allows further orthopedic treatment of patients after 4-5 months without additional bone grafting operations.
\end{abstract}

Keywords: case report, fracture, mandible, osteosynthesis, mini-plate, titanium nickelide, collagenic xenograft

\section{Introduction}

In clinical cases, when the teeth are removed from the fracture line, and the bony defect is not filled, the risk of developing purulent inflammatory diseases increases.

The bony tissue can regenerate and change its micro- and macrostructure. Due to the delicate balance between the formation and loss of bone tissue, changes occur in the form of static and dynamic stress applied to the bone; if the applied stress is greater than the normal physiological level, the equilibrium is deflected towards osteoclasis (this relationship is known as Wolf's law of restructuring of bone structure). Nature has envisioned different types of mechanisms for bone repair after fractures to cope with the different mechanical environments surrounding the fracture. It is known that incomplete fractures (cracks), which allow only micro-movement between fracture fragments, heal with a small number of calluses along the fracture line or without them (primary healing). Unlike incomplete fractures, complete fractures are unstable and therefore generate macro displacement and heal with a large callus protruding from the sides of the bone (secondary tension) [1].

L.N. Hench (2007) in his work identified 4 biomechanical stages of bone restoration after fracture [2-4]:

$1^{\text {st }}$ stage: At the site of the initial fracture, the bone does not function, it is characterized by low rigidity, like that of rubber 
$2^{\text {nd }}$ stage: The bone is not functioning at the site of the original fracture, but there is hard tissue with great rigidity

$3^{\text {rd }}$ stage: The bone is partially non-functional at the site of the original fracture and partially - on the unaffected part of the bone, there is a hard tissue with high rigidity

$4^{\text {th }}$ stage: The site of failure does not correspond to the site of the original fracture. Failure occurs at high rigidity

These changes correspond to cellular changes, depending on time and on the type of extracellular matrix at the site of tissue repair. The strength of the healing fracture increases significantly when mineralization of the osteoid occurs, approximately 4-6 months after the onset of healing.

The choice of treatment helps prevent possible displacement of the bone fragments, which can delay or interfere with the healing of the fracture.

\section{Patient information.}

\section{Materials and Methods}

Patient E., 39 y.o., was admitted to the Department of Maxillofacial Surgery of the Sechenov University with complaints of pain on the left side of the mandible, swelling of the left cheek, limited mouth opening and malocclusion.

Extraoral physical exam revealed altered facial configuration due to soft tissues edema at the left buccal, parotid, and submandibular regions. The skin color was physiological. Pain and fragments mobility at the left mandibular angle were noted during palpation. Regional lymph nodes were not enlarged. The mouth opening was less than 20 $\mathrm{mm}$. There was no evidence of dysphagia. During intraoral checkup lacerations and swelling of the mucosa in the area of the left third lower molar was noted, the crown of the tooth was grossly decayed. Swallowing is free, painless. The occlusion was significantly altered due to fragments dislocation.

The orthopantomogram showed a fracture line in the area of tooth 3.8 and ovalshaped area of radiolucency with teeth 3.7 and 3.8 roots involvement (Fig. 1).

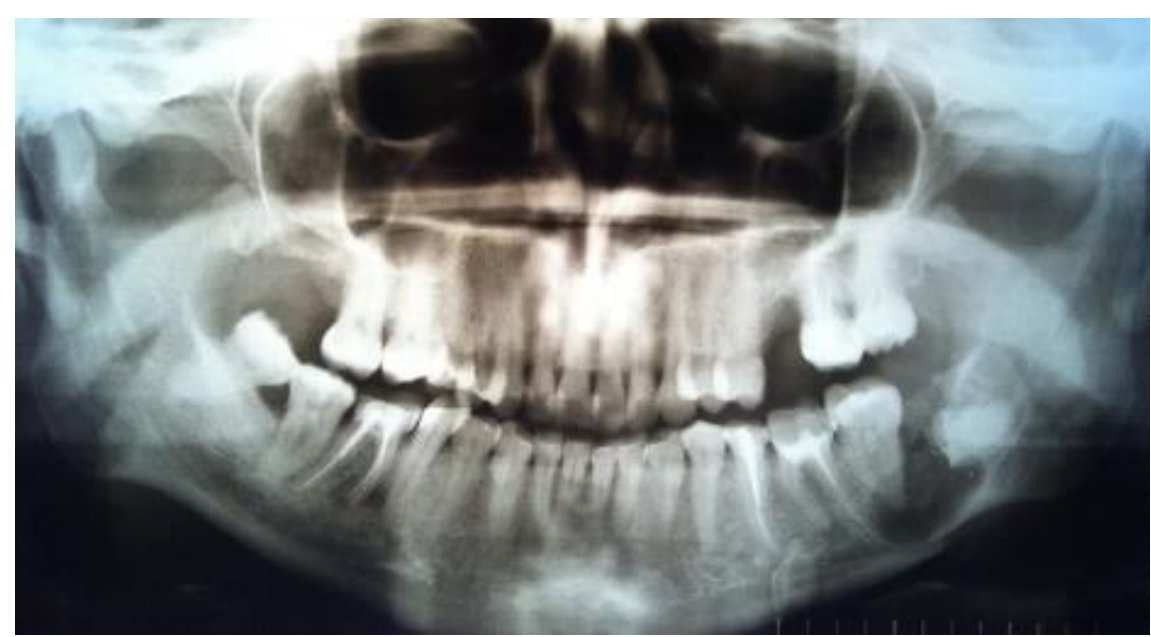

Figure 1. Orthopantomogram before surgery

After primary checkup and radiological evaluation an intermaxillary fixation was performed, the bite was stabilized in a physiological occlusion with arch bars and rubber elastics. 
The patient was examined in a standard pre-surgical volume: laboratory (blood and urine tests) and instrumental (ECG, chest x-ray) studies.

Computed tomography of the lower jaw determined an oval-shapes bony lesion with dimensions of $20 \times 16 \times 16 \mathrm{~mm}$ at the level of teeth 3.7 and 3.8 roots. The bone was swollen at the level of 3.6-3.8 teeth, a periosteal reaction was noted, the contours of the jaw were enlarged, the cortical plates were thinned. A pathological fracture of the lower jaw with fragment displacement occurred, the fracture line passed through 3.7 and 3.8 teeth sockets (Fig. 2).

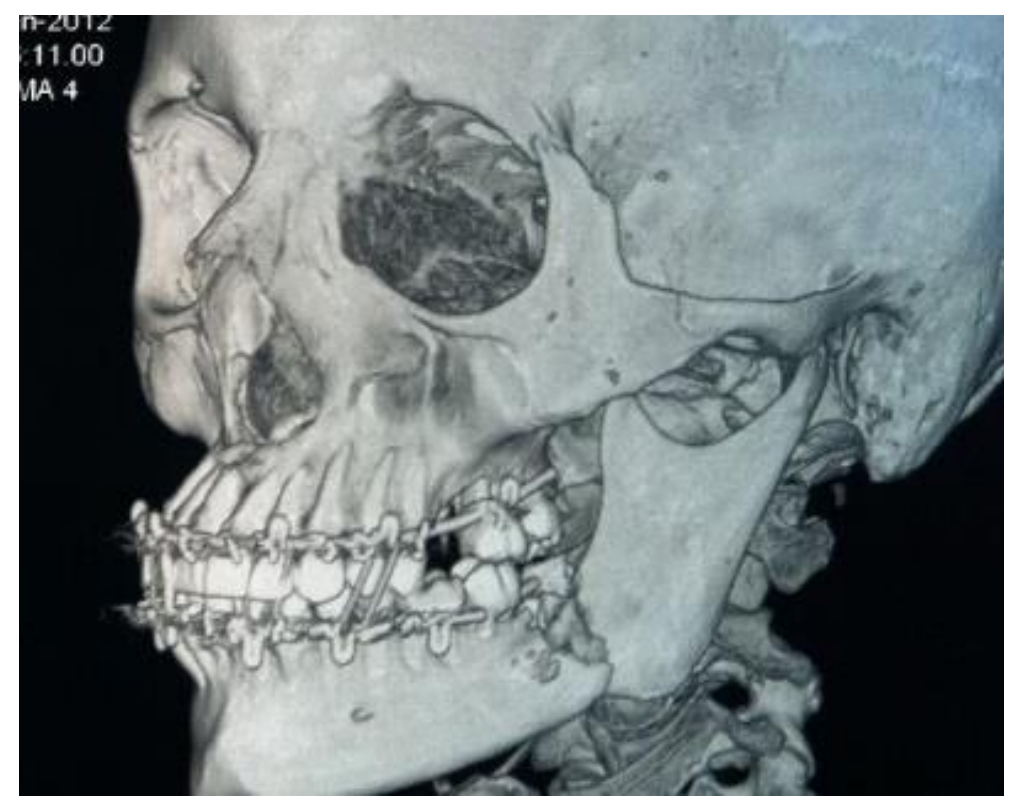

Figure 2. Patient's CBCT at admission

Under general anesthesia (nasotracheal intubation) the patient underwent teeth 3.6, 3.7, 3.8 extraction, cystectomy and osteosynthesis. The angular fracture of the lower jaw was reduced and stabilized with two 6 hole titanium bone mini-plates and $10 \mathrm{~mm}$ length mini-screws. The bony defect was filling with xenograft "Collost". The external approach was chosen due to the violation of the integrity of the mucosa in the area of the fracture line for a long time, the need to remove the cyst, reposition the fragments of the lower jaw and fix them

The patient was treated with the "Collost" osteotropic material (xenogenic collagen osteotropic material obtained from the skin of cattle ("BIOPHARMHOLDING", Moscow, Russia).

\section{Results}

Operation technique.

Stage 1. The teeth 3.6, 3.7 and 3.8 were removed (Fig. 3). 


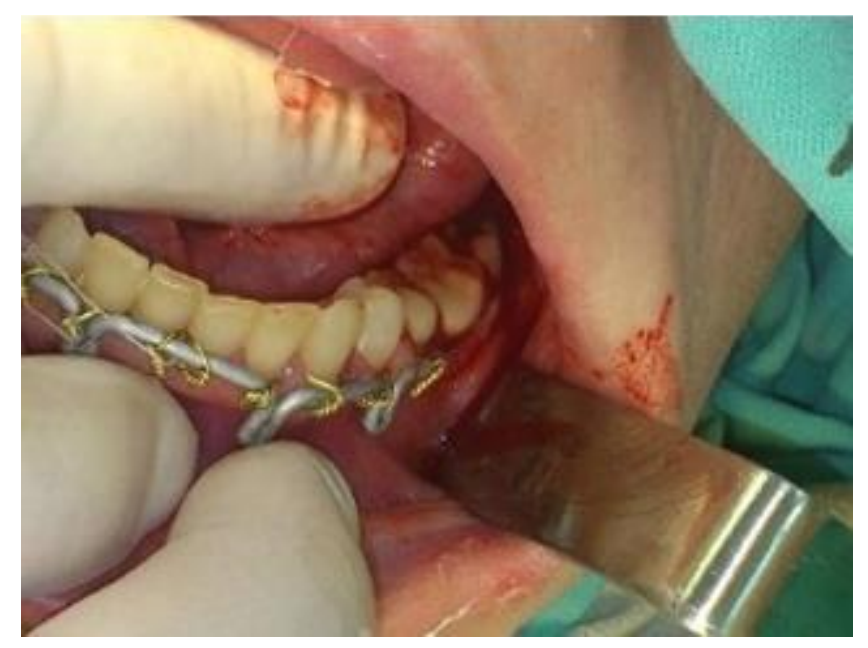

Figure 3. Teeth 3.6, 3.7, 3.8.

A bony cyst 20x15 mm size was determined. A cystectomy was performed and with the removal of small bony fragments $\mathrm{d}=3^{*} 5 \mathrm{~mm}$. (Fig. 4).

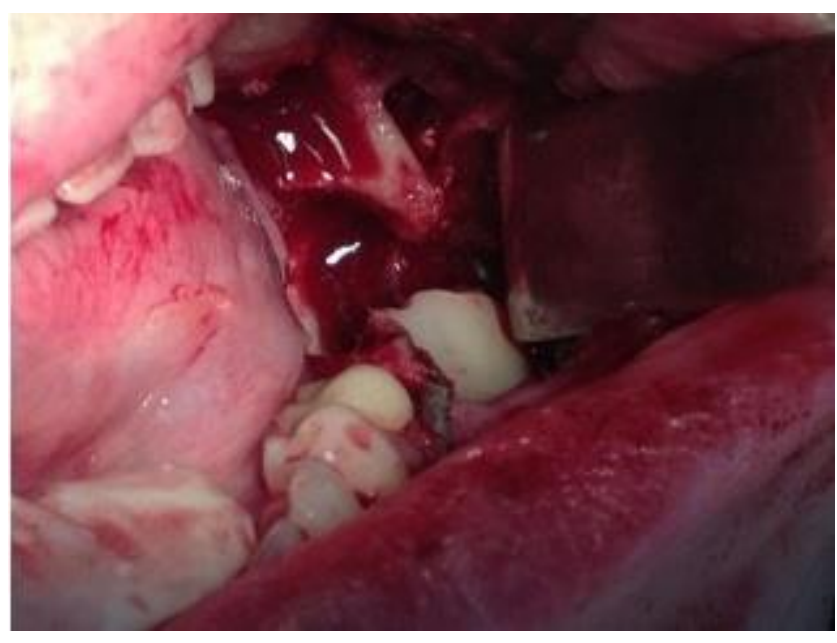

Figure 4. Discovered bone cavity and fracture line

Stage 2. After preliminary marking, an incision was made in the submandibular region on the left, departing from the edge of the lower jaw by $2.0 \mathrm{~cm}$, up to $2.5 \mathrm{~cm}$ long. The skin, subcutaneous fatty tissue is dissected in layers, m. platysma, the own fascia of the neck. The body and the angle of the lower jaw on the left were skeletonized; a planar finely comminuted oblique fracture with a displacement of a smaller fragment upward and inward with a defect in the external cortical plate was found (Fig. 5). 


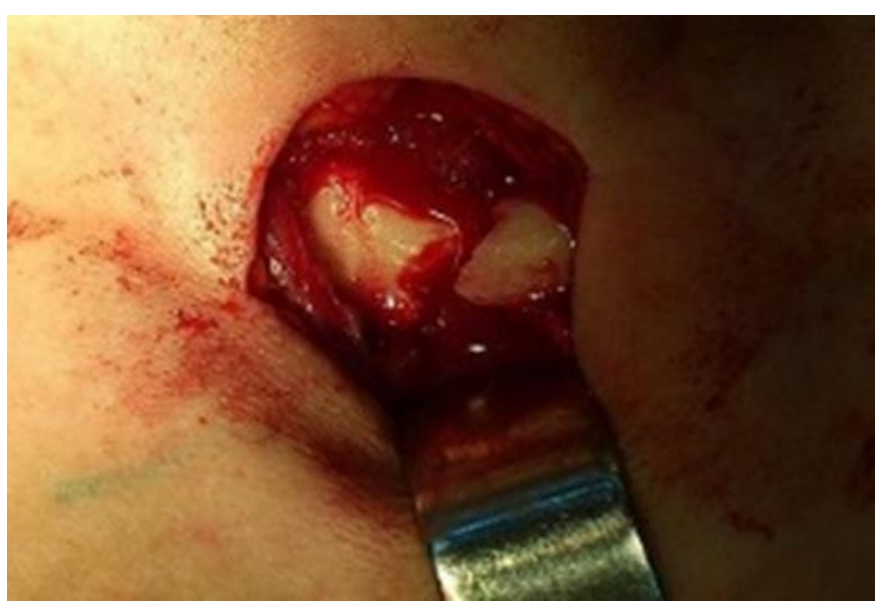

Figure 5. Fracture line visualization

The fracture was reduced, bony fragments were stabilized in a correct position with two titanium 6 hole bone mini-plates and $10 \mathrm{~mm}$ titanium mini-screws (Fig. 6 ).

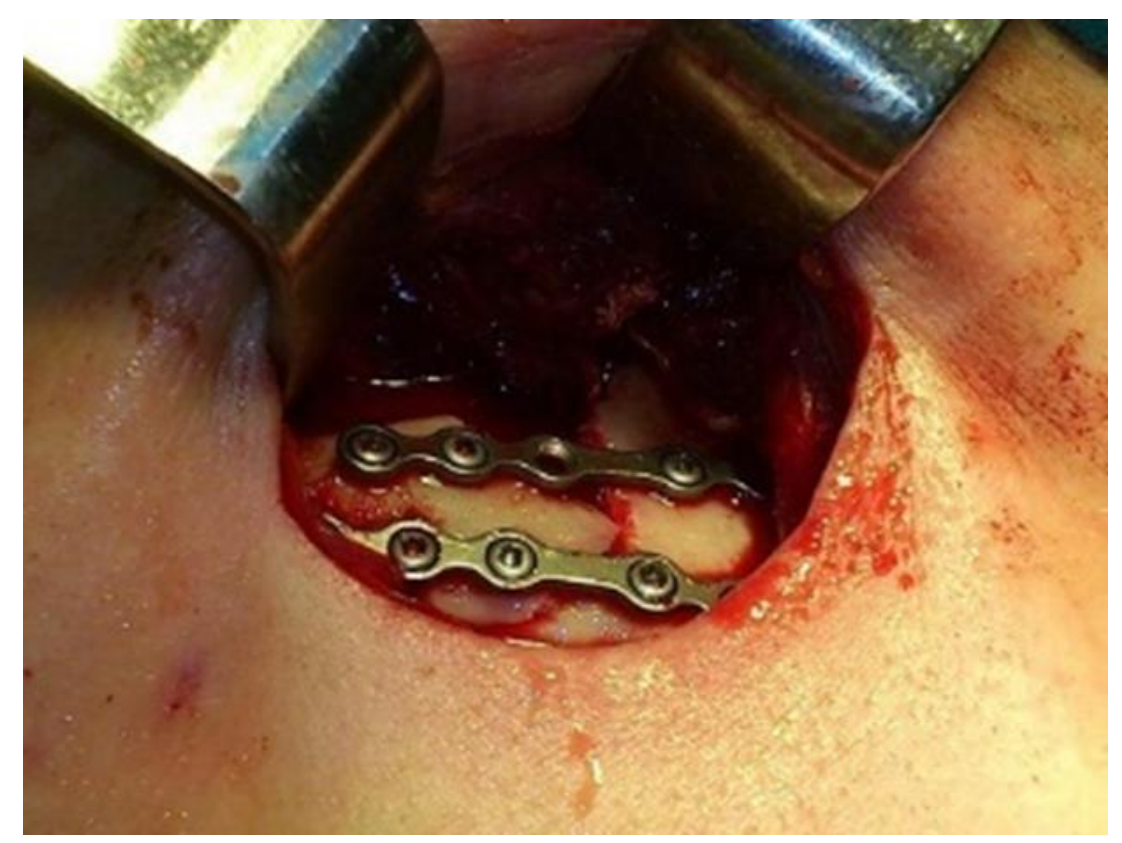

Figure 6 .Osteosynthesis

The wound was washed with antiseptic solutions, layer-by-layer sutured with interrupted sutures with Vicril 3.0 threads. Intradermal suture was applied Prolene 5.0. A plastic catheter was placed at the fracture line (Fig. 7). 


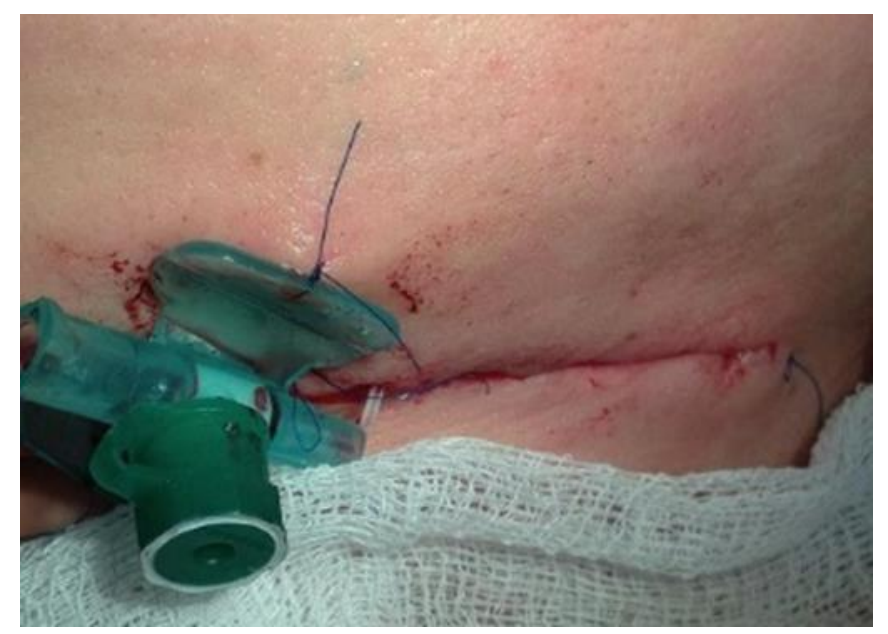

Figure 7. Sutured skin after the intervention

Stage 3. The intraoral operative wound was irrigated with antiseptic solutions. The bone cavity was filled with collagen osteotropic material in a shape of balls and threads. The graft was covered with a resorbable (Fig. 8).

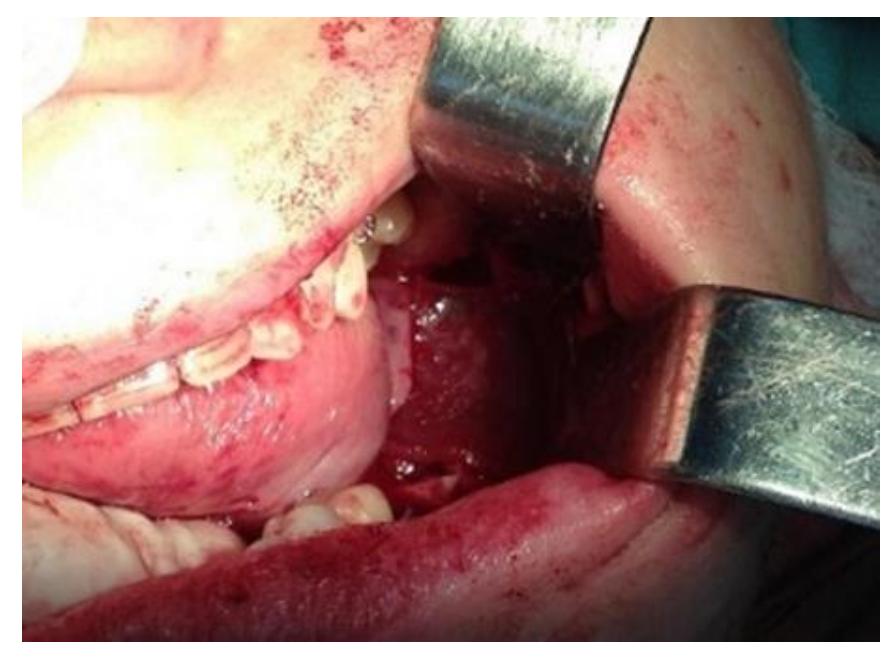

Figure 8. The membrane covers the graft and bony defect.

The wound was sutured with Prolene 4.0 interrupted sutures (Fig. 9).

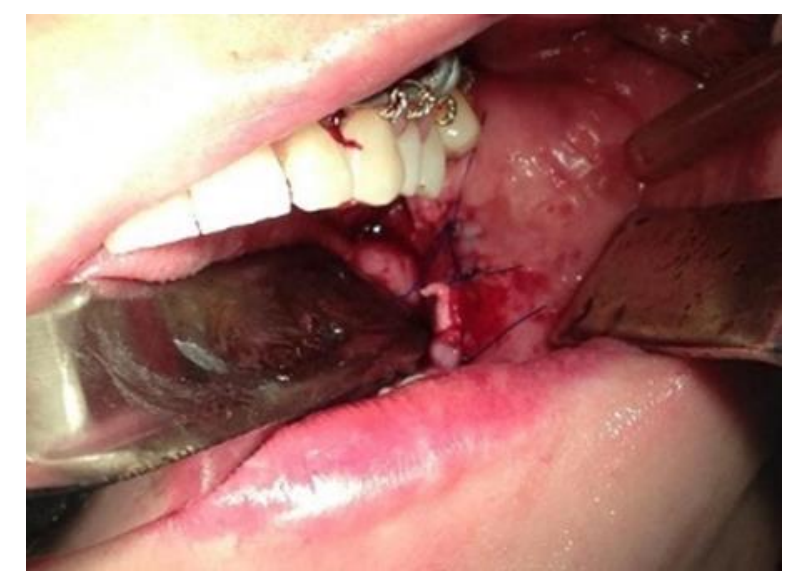

Figure 9. Imposed interrupted sutures in the area of the postoperative wound 
Hemostasis was performed during the surgery. The cyst walls were sent for histological analysis. The results of intraoperative histological examination are as follows: follicular cyst remains.

The postoperative period was uneventful. A course of antibacterial, anti-inflammatory, analgesic therapy was carried out. Daily checkups were performed with intraoral antiseptic solution irrigations. $s$ with, bringing the antibiotic " Lincomycin " to the fracture line through a catheter (Russia, Moscow LLC "Production of medicines") 2 times a day, $900 \mathrm{mg}$.

In the postoperative period the protocol of antibiotic therapy included: cefotaxime 1,0 $\mathrm{g}$ x 2 times per a day intramuscularly, antihistamine (suprastin 1 tablet 1 time per a day), daily dressings, $0,05 \%$ water chlorhexidine rinses 3 times per a day. All treatment was performed for 7 days.

In early postoperative period a control panoramic view was made. The bony cavity after teeth extraction and cystectomy was visualized, titanium plates and screws in a right position. As well as bony fragments were in a correct position (Fig. 10).

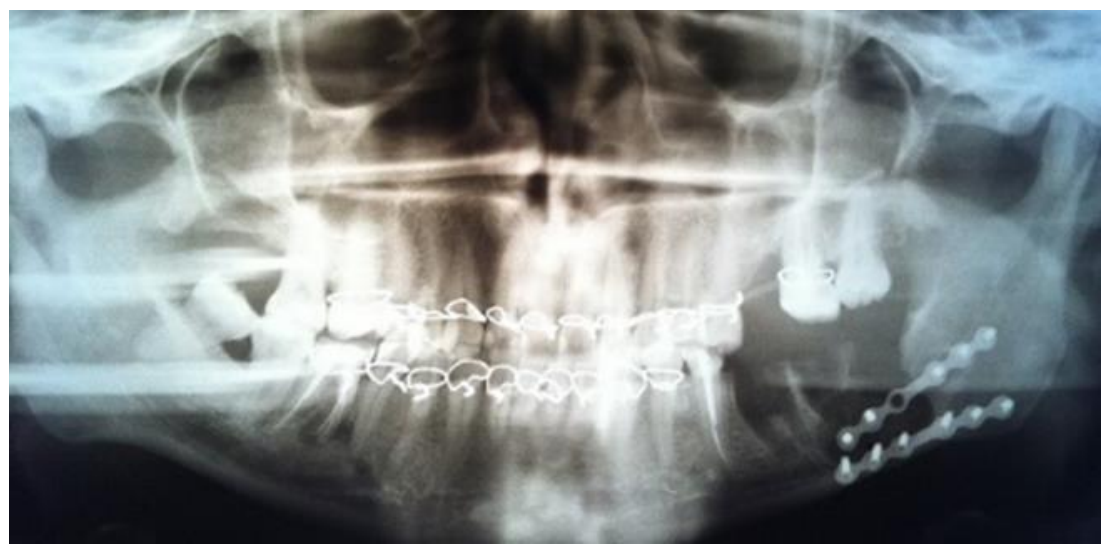

Figure 10. Ortopantomogram after operation

After 7 years the bone reparation process has finished. Furthermore, the patient had dental implants placement. Loss of dental implants is not related to a fracture that occurred more than 7 years ago. Due to improper loading on the installed orthopedic structures, bone resorption occurred (fig.11). 


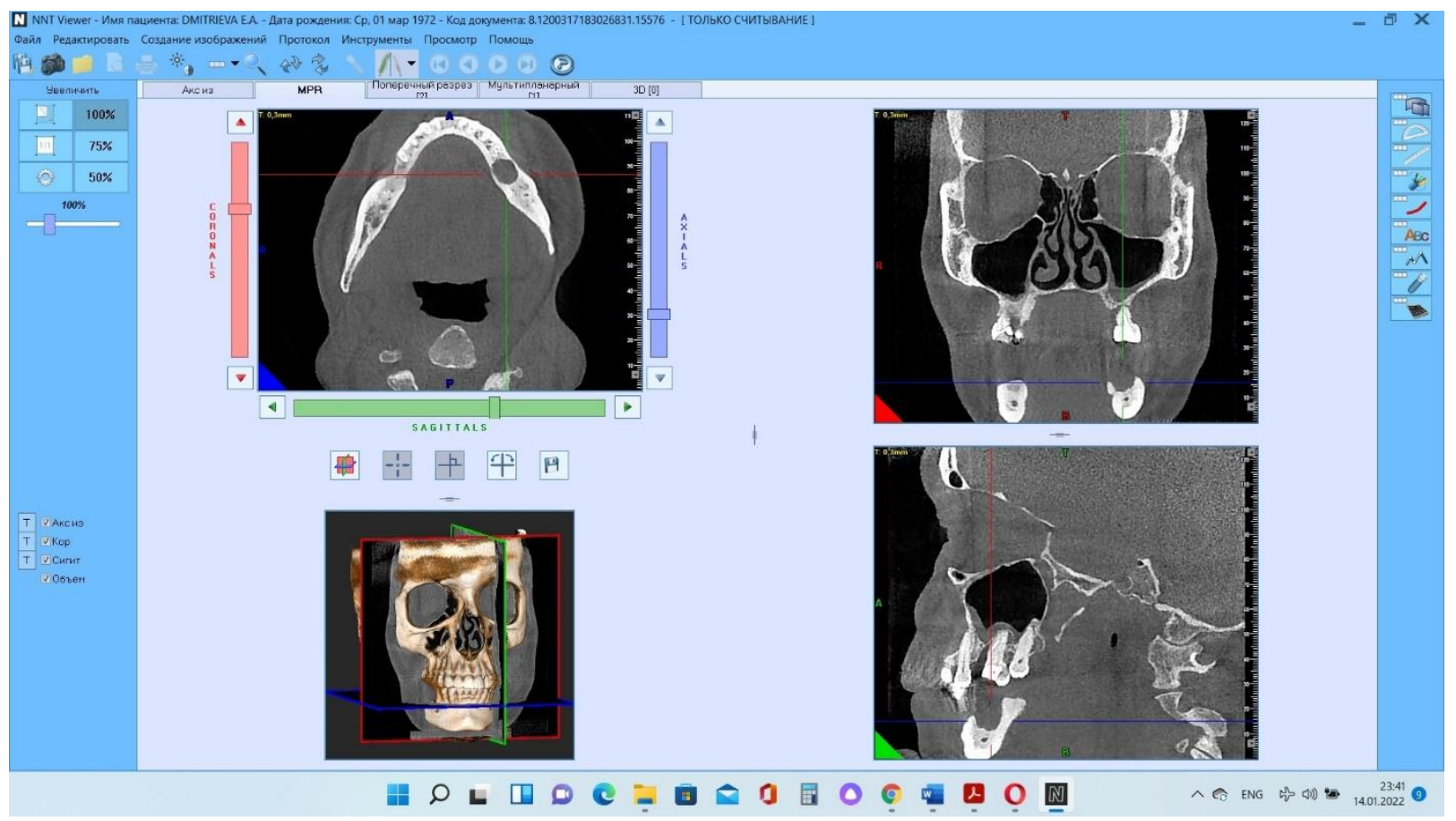

Figure 11. CT-scan after dental implant loss before bone plasty after 7 years surgery

After the removal of the dental implants that have undergone peri-implantitis, repeated CBCT examinations and repeated prosthetics will be performed, considering the mechanical load and the peculiarities of the bite.

\section{Discussion}

The presented articles [5-11] describe the treatment of fractures of the mandible by various modern methods. In all these cases, the technique of bony defect filling with a collagen osteotropic material was used, but the timing of full regeneration of bone tissue was not indicated. which allows us to draw preliminary conclusions about the positive effect of the osteotropic material in this study on the timing of full bone regeneration. Currently the mechanisms of bony regeneration are well studied and may have several factors that affect the outcome of regeneration [12]. But, despite the prevention and treatment, the bone tissue can be restored in full volume not in all cases. That's why xenogenic materials are used to accelerate the reparative processes [13]. The basics of stimulation of regeneration are laid in induction, conduction, and regeneration due to the introduction of osteoprogenic cellular elements (stimulation by substitution). Every potency can be used to stimulate reparative osteogenesis both independently and in combination [14].

In our study, a patient with a fracture of the mandible within the dentition was under observation for 8 years. She performed filling of bone defects with xenogenic bone material. The results of our study indicate a decrease in the timing of osteoreparative processes and rehabilitation when using collagen osteotropic material.

With the use of osteotropic collagen material during the treatment of a patient with this clinical case, the period of healing and regeneration of bone tissue decreased from 6 to 5 months. No inflammatory processes were observed during treatment.

However, in clinical practice, avoidance by a dental surgeon or maxillofacial surgeon of filling the sockets of extracted teeth with osteotropic materials, especially when the fracture line passes through the dentition, can lead to the development of an empty socket 
syndrome, infectious and inflammatory complications, including post traumatic osteomyelitis of the lower jaw. In addition, according to our data, the use of an osteotropic material is also possible in cases of combined pathology of the lower jaw, when, because of surgical intervention, a rather large bone defect is formed, which requires filling, as in the case of our clinical example.

The use of an osteotropic material with osteoconductive and properties can improve the treatment of patients with mandibular fractures within the dentition. This is due to both the stabilization of the fracture line, a decrease in the likelihood of displacement of fragments along with fixation with devices, and a reduction in the time of bone tissue regeneration, which reduces the rehabilitation period and allows further orthopedic treatment of patients in 4-5 months without additional bone grafting operations. The acceleration of the bone reparative process allowed the patient to install dental implants in the area of the missing teeth after 8 years.

\section{Conclusions}

Osteotropic materials of properties can reduce the healing time of bone tissue in the area of fractures.

Author Contributions: “Conceptualization, C.I. and A.L.; methodology, P.P..X.; investigation, E,.D.; resources, E..D.; data curation, C.I.; writing-original draft preparation, E.D.; writing-review and editing, C.I, L.A.;; visualization, S.P.; project administration, S.P. All authors have read and agreed to the published version of the manuscript.

Funding: This research received no external funding.

Institutional Review Board Statement: The study was conducted in accordance with the Declaration of Helsinki and approved by the Institutional Ethics Committee of Sechenov University (protocol code 03-12, date of approval 28/11/2012).

Informed Consent Statement: Informed consent was obtained from a patient.

Data Availability Statement: Data is contained within the article

Acknowledgments: We wish to express our appreciation to rector of the I.M. Sechenov First Moscow State Medical University (Sechenov University) Petr VitalievichGlybochko. The research is supported within project 'Prioritet-2030'

Conflicts of Interest: The authors declare no conflict of interest

\section{References}

1. Sheen JR, Garla VV. Fracture Healing Overview. [Updated 2021 May 12]. In: StatPearls [Internet]. Treasure Island (FL): StatPearls Publishing; 2022 Jan-. Available from: https:/www.ncbi.nlm.nih.gov/books/NBK551678/

2. Reshetova EA et al. Obtaining and studying the properties of composite materials based on zinc-modified hydroxyapatite and biodegradable polyesters. In Proceedings of Perspectives fundamental sciences development, Russia, Tomsk, 21-24 April 2020, Available at http://vital.lib.tsu.ru/vital/access/manager/Repository/koha:000563505

3. Mikhailyuta A.G., Sterleva E.A., Subbotin I.G. Calcium-phosphate materials as biomaterials used in eliminating vicious defects. Alley of Science 2020, Vol. 1, No. 8, pp. 79-84.

4. Balazic, M., Kopac, J., Jackson, M. J., \& Ahmed, W. Titanium and titanium alloy applications in medicine. International Journal of Nano and Biomaterials 2007, Vol. 1, Iss.1, pp.3-34. 1.

5. Bohluli B. et al. Treatment of mandibular angle fracture: Revision of the basic principles. Chinese Journal of Traumatology 2019, Vol . 22, Iss. 2, p. 117-119

6. Bohner L. et al. Treatment of Mandible Fractures Using a Miniplate System: A Retrospective Analysis. Journal of Clinical Medicine 2020, Vol . 9, No. 9, p. 2922.

7. Efimov YV et al. Treatment of patients with unilateral oblique fracture of the lower jaw. Sciences of Europe 2018, No. 24-1 (24), pp.38-42

8. Han C. et al. New surgical instrument for the treatment of condylar fractures: the digitized condylar retractor. British Journal of Oral and Maxillofacial Surgery 2020, Vol. 58, No. 4, pp. 432-436. 
9. Sylvestre A.M. Fracture Management for the Small Animal Practitioner. In book Fractures of the Jaw, pp. 251-268G doi:10.1002/9781119215950.ch24

10. Ambarjan G. M., Karjagdyev S.H. Analiz sushhestvujushih metodov lechenija perelomov nizhnej cheljusti. In Pro-ceedings of Aktual'nye problem razvitija cheljustno-licevoj hirurgii na territorii Povolzh'ja, Russia, Penza, 26/11/ 2020, pp.46-51. Available at http://elib.pnzgu.ru/library/1606391059

11. KhakimovA .A . Comparison of methods for treating fractures of the lower jaw in the dentition. A new day in medicine 2020, No. 2, pp. 587-588

12. Stock, S. R. The Mineral-Collagen Interface in Bone. Calcification Tissue International 2015, Vol. 97, Iss. 3, pp. 262-280.

13. Thompson E. M., Matsiko,A. Farrell E., Kelly D.J., O'Brien F.J. Recapitulating endochondral ossification: a promising route to in vivo bone regeneration. Journal of Tissue Engineering and Regenerative Medicine 2015, Vol. 9, Iss, 8, pp. 889-902.

14. Torreggiani, E., Matthews B.G., Pejda S., Matic I., Horowitz M.C., Grcevic S., Kalajzic I. Preosteocytes/osteocytes have the potential to dedifferentiate becoming a source of osteoblasts. PLoS ONE 2013, Vol. 8, Iss.9, e75204. 